\title{
An estimation algorithm of inserting time-delay in correlation peak to increase location precision of four-element-array
} ZHENG Chenyao $^{1, a}$, Dong Zhenjie ${ }^{2, b}$, XING Qinglong ${ }^{3, c}$, Wu Helong ${ }^{4, d}$

\author{
93 unit of 91388 Army, Zhanjiang, Guangdong, China
}

a zhchy@foxmail.com, ${ }^{\mathrm{b}} 273458057 @ 163 . c o m,{ }^{\mathrm{C} x q l @ 163 . c o m,}{ }^{\mathrm{d}}$ qingwu848@yeah.net

Keywords: four-element-array; time-delay; location precision; correlation peak.

\begin{abstract}
Ultra-short baseline system (USBL) is widely used in ocean observation as a result of small aperture size and ease install. However, the USBL has lower accuracy at a long-range. In order to increase the precision, the estimation algorithm of inserting time-delay in correlation peak is used for passive location four-element-array. Simulation shows that the algorithm can exactly estimate the time-delay difference and increase location precision.
\end{abstract}

\section{Introduction}

USBL system is to simplify the 1970s underwater acoustic location system and developed. It is characterized by small Array dimensions which can be used on a smaller carrier. Its size is only a few centimeters to several tens of centimeters. USBL system is More flexible and convenient to use and easily installs or dippes on the smaller location by carrier selection. USBL acoustic location system has been widely used.

Since the size of hydrophone arrays is small, the use of conventional pulse envelope detection and relative arrival time measurement method in short baseline system will bring a greater error, which requires a new method of measuring and location solver. This paper adopts the algorithm of interpolating time-delay estimation in correlation peak to position USBL which is comparing to the algorithm of cosine curve fitting and polynomial fitting. The simulation result shows that the location accuracy is obviously improved, the two fitting methods has no significant difference, the location error decreases with the SNR increasing and minishes with the array element spacing enlarging. USBL system achieves a high-precision remote location.

\section{Structure of four-element-array location}

The model of four-element-array position, as shown in figure 1.

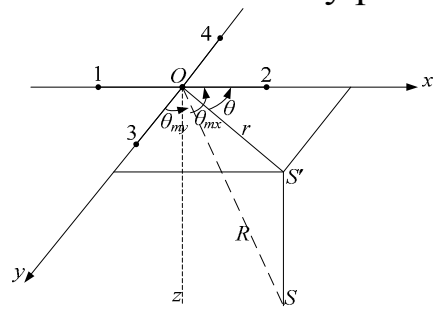

Fig.1 Structure of four-element-array location

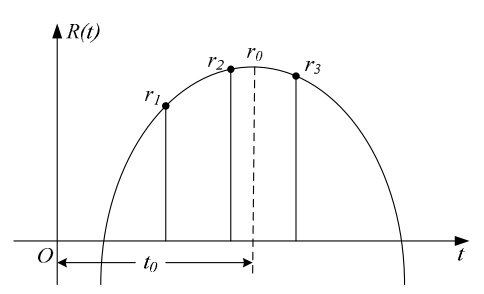

Fig.3 Insertion in correlation peak

$1,2,3,4$ are four hydrophones in figure 1,1 and 2, 3 and 4 are spaced by L, the target is located at $S(x, y, z)$, the target vector-radius is $O S, R$ is the distance between target and array center, $S$ ' is a projection in the $x o y$-horizontal-plane, a included angle $\theta$ with $x$ - axis is horizontal direction angle, $\theta_{m x}, \theta_{m y}$ is separately the angle between $O S$ vector-radius and $x$-axis, $y$-axis. $\tau_{12}$ is a time-delay of target signal between elemnt 1 and 2, $\tau_{34}$ is a time-delay of target signal between elemnt 3 and 4 . When the target position is to meet remote condition in the plane-wave model, in accordance with spatial geometric relations there is: 
$x_{L}=R \cos \theta_{m x}=c \tau_{12} R / L, y_{L}=R \cos \theta_{m y}=c \tau_{34} R / L$.

Where, $c$ is the sound speed underwater, $R$ can be measured by echo-range method. Therefore, the location accuracy depends on the measurement precision of time-delay $\tau$, that is to say an accurately measurement of time-delay is the key to improve location precision.

\section{The algorithm of interpolating time-delay estimation in correlation peak}

Traditional time-delay estimation algorithms have a lower resolution after discrete sampling, a new technique of interpolating in correlation peak $^{[1]}$ can improve the precision of time delay estimation.

\section{Correlator}

We can use the match-filtering method to get the maximum output-peak-power signal to noise ratio(SNR) in the background of white-noise for USBL location system. Since the output of the match-filter is a "panoramic Graphics" of correlator which can be implemented by a cross-correlator. The cross-correlator is a time-compress correlator ${ }^{[2]}$, as shown in Fig 2.

$$
\text { Input } \rightarrow \underset{\text { multiplier }}{\text { Treferance }} \rightarrow \text { integrator } \rightarrow \text { output }
$$

\section{Fig. 2 Correlator}

An input of the correlator is the receive signal (with noise), the other input is the transmitting signal. Within each sampling interval, the reference signal correlative operates with input signal. For example, we can observe the output of correlator by linear frequency modulation (FM) signal which will be transmitted into input of correlator through ideal-channel. If the time-delay of receive signal is $t_{0}$, then the input signal is $S_{i}(t)$ and the reference signal is $S_{r}(t)$ as follows:

$$
s_{i}(t)=A \sin \left(\omega\left(t-t_{0}\right)+\pi \beta\left(t-t_{0}\right)^{2}\right), t_{0} \leq t \leq T+t_{0} ; 0, \text { elses }_{r}(t)=A \sin \left(\omega t+\pi \beta t^{2}\right), 0 \leq t \leq T ; 0, \text { else. }
$$

Where, $\beta=B / T$ is called FM rate, $B$ is the signal bandwidth, $T$ is the signal width. The output of the correlator is ${ }^{[3]}$ :

$$
R_{\text {out }}(t)=A^{2} T /\left[\pi \beta\left(t-t_{0}\right)\right] \sin \left[\pi \beta\left(t-t_{0}\right)\left(1-\left|t-t_{0}\right| / T\right)\right] ; \cos \left[\omega\left(t-t_{0}\right)\right],\left|t-t_{0}\right| \leq T ; 0, \text { else. }
$$

When $t=t_{0}$, the output of the correlator reaches a maximum. If we can detect the output peak of the correlator, the detected peak of output copies, the time-delay $t_{0}$ of the receiving signal can be estimated. Since within each sampling interval, the correlator output is a discrete sample of correlation sequence and therefore the maximum-output cannot be exactly corresponding to the correlation peak leading to measurement error of time-delay. Clearly, the time-delay precision depends on the sampling frequency in the most unfavorable conditions by $1 / 2$ sampling period. In order to obtain higher angle measurement accuracy and thereby get a higher location precision, we must possess a sufficiently high estimation accuracy of time-delay estimation.

\section{Cosine curve fitting}

Correlation function sequence has the form of cosine of quasi-cosine function. We can obtain accurate second of correlation peak by inserting cosin function with this feature ${ }^{[4]}$.Setting fitting waveform function is as follows:

$$
r(\tau)=A_{r} \cos \omega \tau+B_{r} \sin \omega \tau \text {. }
$$

In equation (4) we can deternine the three unknown parameters $A_{r}, B_{r}, \omega$ by another three samples in in order to confirm the cosine signal, as shown in figure 3 . From this the time $t_{0}$ can be obtained which is corresponding to the maximum of correlation peak.

According to this idea in figure 3, we assume to obtain a maximum $r\left(\tau_{2}\right)=r_{2}$ using the peak 
selector, the two adjacent samples before or after $r_{2}$ is $r\left(\tau_{1}\right)=r_{1}$ and $r\left(\tau_{3}\right)=r_{3}$, then:

$$
r_{1}=r\left(\tau_{1}\right)=A_{r} \cos \omega \tau_{1}+B_{r} \sin \omega \tau_{1}, r_{2}=r\left(\tau_{2}\right)=A_{r} \cos \omega \tau_{2}+B_{r} \sin \omega \tau_{2}, r_{3}=r\left(\tau_{3}\right)=A_{r} \cos \omega \tau_{3}+B_{r} \sin \omega \tau_{3} .
$$

By equation (5) solving:

$$
A_{r}=\left(r_{2} \sin \omega \tau_{1}-r_{1} \sin \omega \tau_{2}\right) /\left(\sin \left[\omega\left(\tau_{1}-\tau_{2}\right)\right]\right), B_{r}=\left(r_{1} \cos \omega \tau_{2}-r_{2} \cos \omega \tau_{1}\right) /\left(\sin \left[\omega\left(\tau_{1}-\tau_{2}\right)\right]\right) .
$$

Substituting equation (6) into equation (5), reorganizing to obtain:

$$
r_{3} \sin \left[\omega\left(\tau_{1}-\tau_{2}\right)\right]=r_{2} \sin \left[\omega\left(\tau_{1}-\tau_{3}\right)\right]-r_{1} \sin \left[\omega\left(\tau_{2}-\tau_{3}\right)\right] .
$$

Because $\tau_{2}-\tau_{1}=\tau_{3}-\tau_{2}=T_{s}, T_{s}$ is the sampling period, so that the equation (7) can be rewritten as:

$$
r_{3} \sin \omega T_{s}=r_{2} \sin 2 \omega T_{s}-r_{1} \sin \omega T_{s}=2 r_{2} \sin \omega T_{s} \cos \omega T_{s}-r_{1} \sin \omega T_{s} .
$$

Solving equation (8) to obtain:

$$
\cos \omega T_{s}=\left(r_{1}+r_{3}\right) /\left(2 r_{2}\right) .
$$

Whereby the estimated frequency of the output signal of the correlator is:

$\hat{f}=\left(1 /\left(2 \pi T_{s}\right)\right) \arccos \left(\left(r_{1}+r_{3}\right) /\left(2 r_{2}\right)\right)$.

The actual requirement peak is $r\left(t_{0}\right)=r_{0}$. Because when $\omega t=k \pi+\phi$ ( $\mathrm{k}$ is a non-negative integer), taking the maximum of equation (6), the corresponding time is $t_{0}$ which we desire, so it is:

$\hat{t}_{0}=(k \pi+\phi) / \omega$.

Also we know $t_{0} \in\left(\tau_{1}, \tau_{3}\right)$, so it can be obtained the range of $k$ which is:

$$
\left(\omega \tau_{1}-\phi\right) / \pi \leq k \leq\left(\omega \tau_{3}-\phi\right) / \pi \text {. }
$$

Though all equations Above, we can estimate the time-delay value $t_{0}$.

\section{Polynomial fitting}

We can also use the method of polynomial fitting to obtain the time-delay $t_{0}$. Though the peak-selector we get a maximum $r\left(\tau_{2}\right)=r_{2}$, the two adjacent samples before or after $r_{2}$ is $r\left(\tau_{1}\right)=r_{1}$ and $r\left(\tau_{3}\right)=r_{3}$, we obtain a parabola by doing the secong order fit to these three known points using polynomial fitting $y=a x^{2}+b x+c$, then request the maximum $r_{0}$ ' of this curve, it is corresponding to the maximum $t_{0}$ ' of time-delay that we estimate.

\section{Algorithm simulation}

This paper selects a linear FM signal as the target location signal.

\section{Simulation Process}

The simulation process of the four-element-array passive location with the estimate algorithm of inserting time-delay in correlation peak is shown in figure 4.Their simulation process consists of four steps: The first step is creating signals from four paths which corresponds to the structure of the four-element-array; The second step is coarse measuring time-delay, including the two modules of correlation and peak-selection; The third step is curve fitting, obtaining the interpolation of the measurement correlation peak which corresponds to two fitting curve form of quasi-cosine and parabola, getting a more exact time-delay $\tau_{1}, \tau_{2}, \tau_{3}, \tau_{4}$; The fourth step is calculating time-delay difference $\tau_{12}, \tau_{34}$, then using equation (3) to obtain the target coordinate. 


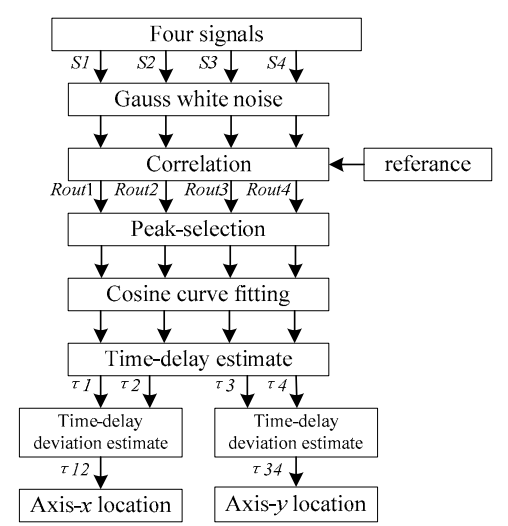

Fig.4 (a) Cosine fitting curve

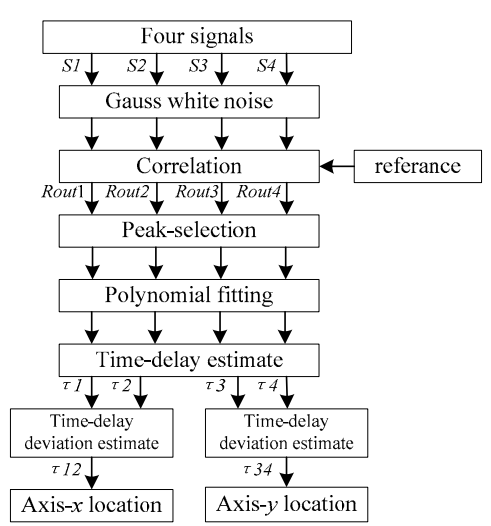

(b) Polynomial fitting curve

\section{Simulation}

Selecting a linear FM signal with a frequency of $12 \sim 15 \mathrm{kHz}$ as the signal of location target, the bandwidth is $B=3 \mathrm{kHz}$, the sampling frequency is $f_{s}=200 \mathrm{kHz}$, the whole pulsewidth is $T=10 \mathrm{~ms}$, the background noise is a band-limited Gauss white noise. The slant range of preset target $S$ is $R=8000 \mathrm{~m}$, the coordinate of $S^{\prime}$ is $(4000,4000)$. Given: the spacing between elment 1 and 2 is $L=320 \mathrm{~mm}$, the speed of sound in water is $c=1500 \mathrm{~m} / \mathrm{s}$.

\section{1) Simulation I : Plane Location solver}

In accordance with the estimation algorithm theory of inserting cosine curve time-delay in correlation peak, After doing 500 times independent statistics respectively using Monte Carlo algorithm when SNR is $10 \mathrm{~dB}$ and $15 \mathrm{~dB}$, we obtain location points of the target in the horizontal plane in figure 5 and figure 6 . Where $x$ and $y$ are the horizontal location coordinates with the elemnt-spacing $L=320 \mathrm{~mm}, \sigma_{L x}$ ' is the mean square deviation which is calculated as:

$$
\sigma_{\mathrm{Lx}}{ }^{\prime}=\sqrt{\left(\sum_{i=1}^{N}\left(x_{i}-x_{0}\right)^{2}\right) / N}
$$

Where, $x_{i}$ is the measurement, $x_{0}$ is the mean of target position projecting to x-axis in horizontal plane, $N$ is the times of statistics.Comparing figure 5 with figure 6 , it is shown that the location deviation of target position is larger and the location points are more dispersive when SNR is 10dB, the location deviation of target position is smaller and the location points are more concentrated when SNR is $15 \mathrm{~dB}$. Though statistics calculating, the location mean square deviation $\sigma_{L x}$ ' are separately $4.38 \mathrm{~m}$ and $2.52 \mathrm{~m}$ when $\mathrm{SNR}$ is $10 \mathrm{~dB}$ and $15 \mathrm{~dB}$. For a four-element array passive location system, using the estimation algorithm of inserting time-delay in correlation peak to exactly measure time-delay deviation will obtain a higher location precision.

Then in accordance with the estimation algorithm theory of inserting polynomial time-delay in correlation peak, we do 500 times independent statistics respectively using Monte Carlo algorithm when SNR is $10 \mathrm{~dB}$ and $15 \mathrm{~dB}$ once again, as shown in figure 7 and figure 8.

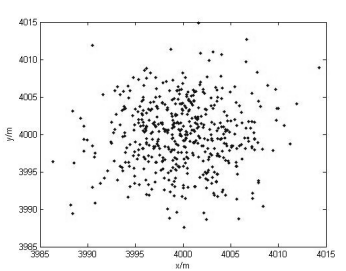

Fig. 5 SNR $=10 \mathrm{~dB}$

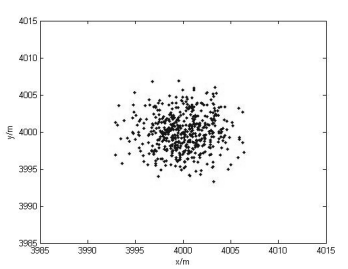

Fig. $6 \mathrm{SNR}=15 \mathrm{~dB}$

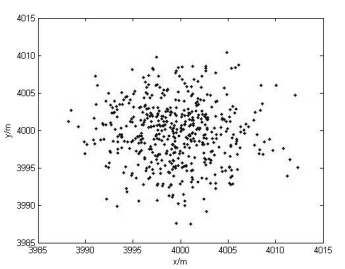

Fig.7 SNR=10dB

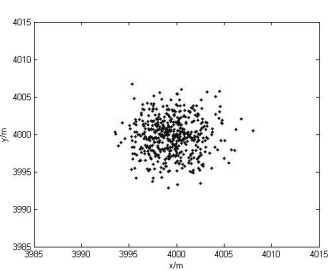

Fig. $8 \mathrm{SNR}=15 \mathrm{~dB}$

Though calculating, the location mean square deviation $\sigma_{L x}$ ' is separately $4.34 \mathrm{~m}$ and $2.44 \mathrm{~m}$. It follows that the result of polynomial fitting is better than the result of cosine curve fitting with the estimate algorithm of inserting time-delay in correlation peak, but the former has a larger operation and requires for a long time. 


\section{2) Simulation II : Comparison of location error in different array element spacing}

USBL location system using the estimate algorithm of inserting three points in correlation peak to achieve 100 independent statistics by Monte Carlo method, we obtain location error as shown in table 1 .

Table 1 Location error in different array element spacing

\begin{tabular}{|c|c|c|c|c|c|c|c|c|}
\hline$L /(\mathrm{mm})$ & 4 & 8 & 16 & 32 & 48 & 64 & 80 & 96 \\
\hline$\sigma_{L x} /(\% \mathrm{R})$ & 2.51 & 1.24 & 0.63 & 0.36 & 0.21 & 0.16 & 0.11 & 0.09 \\
\hline
\end{tabular}

Table 1 shows that the location error is on the decrease along with increase of array element spacing. That is to say USBL location system permillage corresponding to slant distance can reach 0.09 by increasing the array element spacing.

According to the location error calculating equation, given a value of $L$, we can obtain error $\sigma_{L x}$ in the axis- $x$. In consideration of increasing array element spacing will bring about installation difficulty and cost increase in the four-element-array passive system, we will split the difference and select an optimal value of $L$.

\section{Conclusion}

This paper uses the estimation algorithm of inserting time-delay in correlation peak to locate USBL in the structure of four-element-array passive location, compares cosine curve fitting with polynomial fitting. Simulation results that we obtain a higher location precision, the two fitting method have non-obvious difference, the location precision is on the decrease along with increase of SNR and array element spacing. USBL system remotely get a high location accuracy.

\section{References}

[1] Huang Jianren.An algorithm of interpolating time-delay estimation in correlation peak[J]. Acoustic Journal,2005.

[2] Tian Tan. Technique of underwater positioning and navigation.National Defense Industry Press, 2007.9.

[3] Tian Tan,Liu Guozhi,Sun Dajun.Technique of sonar[M].Harbin:Harbin Engineering University Press, 2006.

[4] Liu Li.Research on technique of underwater acoustic communication[D].Harbin:HEU Press, 2006 